\title{
Análisis de las alteraciones posturales ocasionadas por la práctica del surf.
}

\section{Analysis of postural changes caused by surfing.}

Pág. 46,51

Recibido: $14-06-2020$

Aceptado: 23-06-2020

Ybi Rojas Arias ${ }^{1}$

Luis Carlos Chacón Sancho²

1. Escuela de Fisioterapia,UCIMED, San José, Costa Rica.

2. Escuela de Fisioterapia,UCIMED,San José, Costa Rica.

\section{RESUMEN}

\section{INTRODUCCIÓN}

El surf es un deporte que se ha popularizado y ampliado alrededor del mundo, de ahí la importancia de conocer las alteraciones que se pueden producir por su práctica.

\section{MÉTODOS}

Se elaboró una revisión sistemática de estudios no experimentales de corte transversal en las bases de datos como: PubMed, Scopus, Annual Reviews, Sport Discus y PEDro, siguiendo los parámetros de la estructura PRISMA.

\section{RESULTADOS}

A partir del análisis de los estudios incluidos se muestran cambios en la postura por la práctica del Surf y se encontró que los deportistas del surf amateurs (recreativos) tienen mayor incidencia en desviaciones posturales versus profesionales. Con relación a las alteraciones posturales la zona más afectada del cuerpo es la región lumbar y entre las alteraciones más comunes de la región superior se encuentran las desviaciones del hombro anterior y lateral.

\section{CONCLUSIÓN}

Se concluye que la práctica del surf crea modificaciones que alteran la postura debido al gesto propio del deporte y que están condicionadas con base al tiempo de práctica y nivel del practicante.

\section{PALABRAS CLAVE}

Surf. Postura. Patologías. Alteraciones. 


\section{ABSTRACT}

\section{INTRODUCTION}

Surfing is a sport that has become popular and expanded around the world, hence the importance of knowing the changes that can occur due to its practice.

\section{METHODS}

A systematic review of non-experimental cross-sectional studies was carried out in databases such as: PubMed, Scopus, Annual Reviews, Sport Discus and PEDro, following the parameters of the PRISMA structure.

\section{RESULTS}

the results of the study changes in position due to the practice of Surfing and it was found that amateur (recreational) surf athletes have a greater incidence in postural deviations than professionals. In relation to postural alterations, the most affected area of the body is the lumbar region and among the most common alterations in the upper region are deviations of the anterior and lateral shoulder.

\section{CONCLUSION}

It is concluded that the practice of surfing creates modifications that alter the posture due to the gesture of the sport and that are conditioned based on the practice time and level of the practitioner.

\section{KEY WORDS}

Surf. Posture. Pathologies. Alterations.

\section{INTRODUCCIÓN}

Actualmente, el surf se ha convertido en una práctica deportiva muy popular y presente en todo el mundo (Inada et al., 2018). Es considerado uno de los deportes más completos a nivel tanto aeróbico por la remada, como anaeróbico durante el tiempo de flow en la tabla (Esparza, 2011; Deamínguez, 2016). Su práctica requiere un gran esfuerzo físico, sustentada principalmente por la acción de los músculos para equilibrar la postura, y que a su vez requiere de un gran balance, fuerza y resistencia muscular para la coordinación (Paillard et al., 2011).

El surf como ejercicio y deporte está asociado a innumerables beneficios, desde la prevención de enfermedades crónico-degenerativas hasta el mejoramiento de la calidad vida (Gaspe, 2015). Toda práctica de ejercicio físico continuo y prolongado conlleva adaptaciones crónicas fisiológicas y biomecánicas indiscutibles. Sin embargo, si las características de la práctica y su ejecución no son óptimas pueden repercutir en efectos adversos, que con el tiempo pasan de un irregular e inofensivo cambio a una posible alteración del cuerpo (Wu, 2018).

Durante la práctica del surf son muchas las fuerzas que influyen en los movimientos, estás son: (a) las intrínsecas asociadas a la contracción muscular y la respuesta nerviosa ocasionada por la necesidad de coordinación y estabilidad, y las (b) extrínsecas, cuyas fuerzas se manifiestan a través del ambiente (el viento y el mar), así como el comportamiento de la tabla sobre la ola. Por lo tanto, si durante la ejecución de los movimientos, la musculatura del cuerpo no se encuentra equilibrada adecuadamente, existe una probabilidad mayor de que aparezcan desbalances musculares a largo plazo que 
modifiquen la postura (Chapman et al., 2008; Freeman et al., 2013).

Por su parte, la aparición de trastornos posturales ha sido evidenciada a lo largo de los años en los deportes, sea a partir de la repetición excesiva de posturas incorrectas, ejecución errónea de gestos técnicos, realización de movimientos involuntarios sostenidos y sobrecargas durante el entrenamiento. Múltiples estudios, han relacionado la práctica del surf con el origen de lesiones y el desarrollo de trastornos posturales (Deamínguez, 2016; Moura et al., 2014; Novoa, 2015; Wu, 2018).

La comprensión de estas deformaciones biomecánicas y posturales permiten identificar las alteraciones de la postura que se pueden producir en las personas que practican surf, y así prevenir, tratar y corregir. Por lo tanto, el propósito de este estudio es sistematizar los efectos de la práctica continua del Surf sobre la postura y el hallazgo de posibles alteraciones.

\section{METODOLOGÍA}

La investigación llevada a cabo es una revisión sistemática de estudios no experimentales siguiendo las pautas del Preferred Reporting Items for Systematic reviews and Meta-Analyses (Moher, Liberatl, Tetzlaff, Altman y PRISMA Group, 2009).

\section{BÚSQUEDA DE LITERATURA}

Se efectuó una búsqueda en bases de datos como: SportDiscus, PubMed, Annual Reviews, Scopus, y PEDro. Se utilizaron los siguientes términos de búsqueda para la revisión: postural alterations in Surf, posture and surf y surf effects on posture.
Figura 1.

Diagrama de flujo del proceso de revisión sistemática de la literatura.

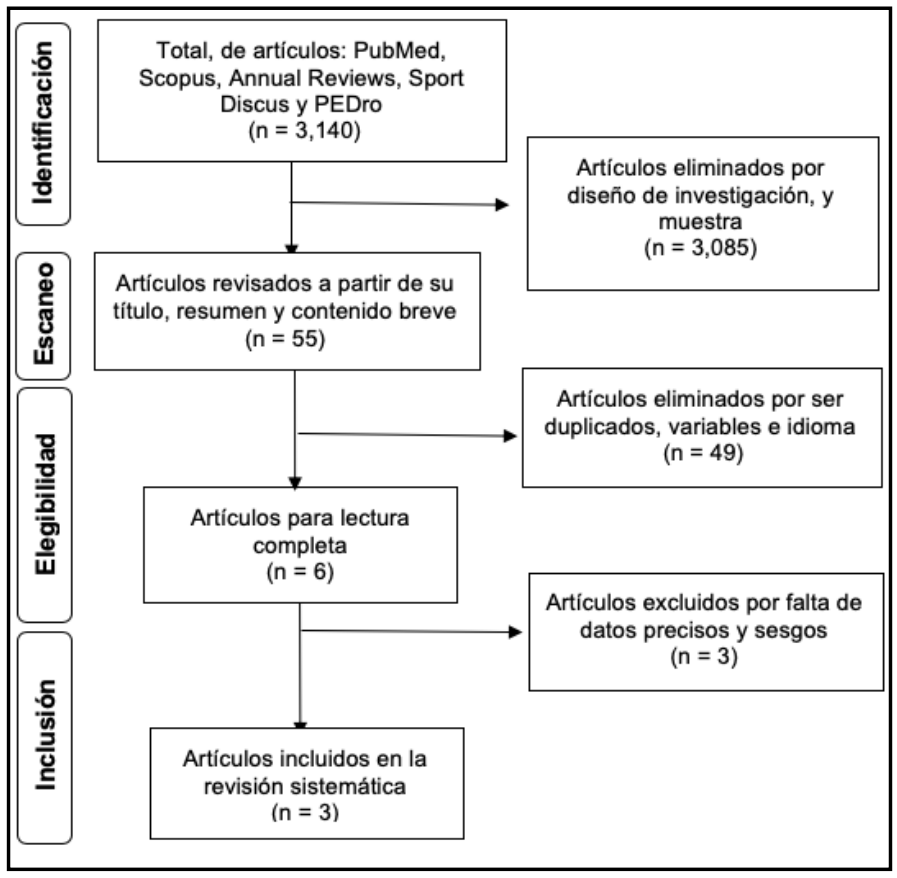

Fuente: Elaboración propia siguiendo las pautas de Moher, D., Liberati, A., Tetzlaff, J., Altman, D. y The PRISMA Group. (2009). Preferred Reporting Items for Systematic Reviews and Meta-Analyses: The PRISMA Statement. PLoS Med, 6(7): e1000097. doi:10.1371/journal.pmed1000097. Simbología: $n=-$ cantidad de estudios.

Los criterios de inclusión y exclusión utilizados para la revisión sistemática de la literatura para esta investigación fueron: (a) estudios no experimentales: transversal y longitudinal; (b) investigaciones con información completa en cuanto a todo el procedimiento y las características de la muestra utilizada para el análisis; (c) resultados que se reportaron como un trastorno o alteración, y no cómo una lesión o trauma, o enfermedad; (d) trabajos desde enero del 2008 hasta abril del 2020. (e) estudios sólo en español, inglés o portugués y (f) muestras con un tiempo de práctica del surf mayor a 1 año continuo. 


\section{ANÁLISIS DE LA INFORMACIÓN Y CALIFICACIÓN}

Para esta revisión, se recogieron los datos de los estudios que informaron sobre las alteraciones que provocaba la práctica del Surf en la postura. Se analizó la relación que había entre las características de la muestra participante para la comparación y discusión sobre la presencia de las alteraciones posturales. En cada investigación que fue incluida en esta revisión, se registraron los datos de: autores y año, tamaño de la muestra ( $n$ ) divididos por sexo, nivel de rendimiento, tiempo y frecuencia de práctica, instrumentos de medición - evaluación, y resultados obtenidos.

\section{RESULTADOS}

Tabla 1.

Sistematización de los resultados de las investigaciones para su análisis

\begin{tabular}{|c|c|c|c|c|c|}
\hline Autor y año & $\mathrm{N}$ & Nivel & Tyf & Instrumentos & Resultados \\
\hline $\begin{array}{l}\text { Deaminguez } \\
\text { (2016) }\end{array}$ & $\begin{array}{l}\mathrm{N}_{\mathrm{h}}: 26 \\
\mathrm{~N}_{\mathrm{m}}: 19\end{array}$ & $\begin{array}{l}\text { Am: } \\
30 \\
\text { Pro: } \\
15\end{array}$ & $\begin{array}{l}\text { T:1 a } 3 \\
\text { f: } 1-3\end{array}$ & $\begin{array}{l}\text { Test de isquiotibiales } \\
\text { (TFT), test del } \\
\text { cuadrado lumbar } \\
\text { (TFT), test de } \\
\text { movilidad del psoas } \\
\text { Leopold Busquet. } \\
\text { Prueba de rascado } \\
\text { de Apple. }\end{array}$ & $\begin{array}{l}\text { El } 26 \% \text { suffió de deshidratación } \\
\text { discal, el } 24 \% \text { hemia de disco, el } \\
21 \% \text { pinzamiento, con el } 17 \% \\
\text { contractura, el } 3 \% \text { tenemos } \\
\text { espondilolistesis, conducto estrecho } \\
\text { y esguince }(3 \%) \text { El gesto deportivo } \\
\text { de la remada fue el de mayor } \\
\text { limitación fue del } 44 \% \text {. }\end{array}$ \\
\hline $\begin{array}{l}\text { Peirao, } \\
\text { Tirloni y } \\
\text { Reiss (2008) }\end{array}$ & $\mathrm{N}_{\mathrm{n}}: 17$ & Pro:17 & $\begin{array}{l}\text { T: } 13.7 \\
\pm 3.9 \\
\text { f: } 4.5 \pm \\
1.4\end{array}$ & $\begin{array}{c}\text { Método (PSU) } \\
\text { Índice de corrección } \\
\text { postural (ICP) }\end{array}$ & $\begin{array}{l}\text { El } 70.6 \% \text { de los atletas presentaron } \\
\text { desviación lateral del hombro. El } \\
70.6 \% \text { aumento de la curvatura } \\
\text { lumbar y } 76.5 \% \text { de las rodillas } \\
\text { hiperextendidas, el indice de } \\
\text { corrección postural (PCl) promedio } \\
\text { fue de } 86.6 \pm 5.0 \% \text { (niveles } \\
\text { considerados buena postura). }\end{array}$ \\
\hline $\begin{array}{l}\text { Gaspe } \\
\text { (2015) }\end{array}$ & $\begin{array}{l}\mathrm{N}_{\mathrm{h}}: \\
23 \\
\mathrm{~N}_{\mathrm{m}}: 7\end{array}$ & Am:30 & $\begin{array}{l}T: 2 \\
f: 2 a 4\end{array}$ & $\begin{array}{l}\text { Goniometría, Test de } \\
\text { acortamiento } \\
\text { muscular; Análisis } \\
\text { visual de la pisada } \\
\text { estática. }\end{array}$ & $\begin{array}{l}\text { En alteraciones posturales, la más } \\
\text { frecuente es la hiperlordosis }(93 \%) \text {,) } \\
\text { la anteversión de la pelvis }(85 \%) ; \text {; } \\
\text { recurvatum de rodilla con un } 96,6 \% \text {; } \\
\text { valgo de rodilla }(53,3 \%) \text { y dentro de } \\
\text { este porcentaje el } 87,5 \% \text { presentó } \\
\text { rótulas divergentes, la alteración a } \\
\text { nivel de la rodilla más frecuente es } \\
\text { el falso valgo; rotación externa de } \\
\text { cadera (60\%); retropié pronado y } \\
\text { altamente pronado ( } 56,6 \%) \text {; dedos } \\
\text { en garra }(88,4 \%) \text { con deformidad } \\
\text { moderada en el } 57 \% \text { de los casos; } \\
\text { deformidad moderada en hallux } \\
\text { valgus del primer dedo del pie; y } \\
\text { apertura de miembros inferiores en } \\
\text { el } 63,3 \% \text { de los casos. }\end{array}$ \\
\hline
\end{tabular}

\section{DISCUSIÓN}

Examinando los resultados de las tres investigaciones se puede observar las diferencias de los deportistas del surf amateurs versus profesionales donde en los deportistas recreativos se indica una mayor incidencia de desviaciones posturales, entre estas alteraciones las más comunes de la región superior se encuentran las desviaciones del hombro anterior y lateral, cabe a resaltar que en otros casos los surfistas aficionados han mostrado incluso ambas desviaciones (Carter et al., 2015; Deamínguez, 2016; Inada et al., 2018).

En cuanto a diferencias ligadas al sexo, no se hallaron diferencias significativas por presencia de alteraciones, tiempo de práctica o nivel de entrenamiento. Por su parte, la literatura actual tampoco brinda más información sobre esta comparación.

Las irregularidades en la alineación del cuerpo, como la hiperextensión de las rodillas, se pueden dar a causa de la ejecución del golpe, ya que durante el movimiento se realiza la hiperextensión de las rodillas para facilitar el equilibrio del surfista sobre la tabla, creando una mala postura y alterándola (Gaspe, 2015; Slayback, 2014).

Fuente: Elaboración propia. Simbología: $\mathrm{N}=$ Cantidad de participantes, $A m=$ Amateur, Pro $=$ Profesional, $T=$ tiempo de práctica en años, $f=$ frecuencia en días de sesiones prácticas, $\mathrm{Nh}=$ cantidad de participantes hombres, $\mathrm{Nm}=$ cantidad de participantes mujeres. 


\section{CONCLUSIÓN}

En relación con las alteraciones posturales de los diferentes artículos se observó que la hiperlordosis es la más frecuente, en sí la zona más afectada del cuerpo es la región lumbar donde también encontramos la deshidratación discal muy común en los surfistas y como tercera patología frecuente está el pinzamiento de disco (Inada et al., 2018; Moura et al., 2014).

También, el gesto propio de la remada durante la práctica del surf es un movimiento que influye sobre la adquisición de una posición anormal e inestable debido a la permanencia en hiperextensión de la cabeza, esta necesidad del deporte de mantener dicha postura sumado a factores de estrés ambiental conllevan a una sobrecarga de la región lumbar y una tensión continuada de los músculos estabilizadores del cuello y espalda (Chapman et al., 2007; Deamínguez, 2016; Frank et al., 2009; Gaspe, 2015).
Se concluye que la práctica del surf crea modificaciones posturales que alteran la postura debido al gesto propio del deporte y que están condicionadas a base del tiempo y sus acciones.

La incidencia de las alteraciones posturales muestra ser mayor en el tipo de surfista amateurs que en los profesionales, debido a que el surf es un deporte que demanda mucho esfuerzo donde se requiere de equilibrio, fuerza muscular, coordinación y concentración; para ello lo mejor es buscar ayuda de un profesional para realizar estas prácticas de manera adecuada. Finalmente, la zona lumbar es la región con mayor incidencia de alteraciones, sin embargo, la misma sólo es frecuente en surfistas amateurs. Por su parte, las alteraciones posturales en surfistas profesionales se dieron más a nivel de cuarto superior enfocadas en la región del hombro. Así mismo las desviaciones posturales en miembros inferiores se da en igual medida para ambos tipos de surfistas, pero en menor medida.

\section{REFERENCIAS BIBLIOGRÁFICAS}

Carter, J., Marshall, N. y Abbott, A. (2015). Shoulder pain and dysfunction in young surf lifesavers. Physical Therapy in Sport, 16(2): 162-168. https://doi.org/10.1016/j.ptsp.2014.10.004

Chapman, D., Needham, K., Allison, G., Lay, B. y Edwards, D. (2008). Effects of experience in a dynamic environment on postural control. British Journal of Sports Medicine, 42(1), 16-21. https://doi.org/10.1136/bjsm.2006.033688

Deamiguez, A. (2016). Patologías de columna vertebral en surfistas. Universidad Fasta. Facultad de Ciencias Médicas http://redi.ufasta.edu.ar:8080/xmlui/handle/123456789/1293

Esparza, D. (2011). De Hawai al Mediterráneo: La génesis del surf en España. RICYDE. Revista Internacional de Ciencias Del Deporte, 7(26): 370-383. https://doi.org/10.5232/ricyde2011.02603 
Frank, M., Zhou, S., Bezerra, P. y Crowley, Z. (2009). Effects of long-term recreational surfing on control of force and posture in older surfers: A preliminary investigation. Journal of Exercise Science and Fitness, 7(1): 31-38. https://doi.org/10.1016/S1728-869X(09)60005-8

Freeman, J., Bird, S. y Sheppard, J. (2013). Literature Review: Surfing performance, injuries and the use of the y balance test. Journal of Australian Strength and Conditioning J. Aust. Strength Cond, 21(2): 32-39.

Gaspe, G. (2015). Las alteraciones posturales en miembros inferiores en el surf. 28/05/20, de Universidad Fasta. Facultad de Ciencias Médicas. http://redi.ufasta.edu.ar:8080/xmlui/ handle/123456789/895

Inada, K., Matsumoto, Y., Kihara, T., Tsuji, N., Netsu, M., Kanari, S., Yakame, K. y Arima, S. (2018). Acute injuries and chronic disorders in competitive surfing: From the survey of professional surfers in Japan. Sports Orthopaedics and Traumatology, 34(3), 256-260. https://doi.org/10.1016/j. orthtr.2018.03.107

Moher, D., Liberati, A., Tetzlaff, J., Altman, D. y The PRISMA Group. (2009). Preferred Reporting Items for Systematic Reviews and Meta-Analyses: The PRISMA Statement. PLoS Med, 6(7): e1000097. doi:10.1371/journal.pmed1000097

Moura, D., Sincari, M. y Fonseca, P. F. (2014). Caso clínico Diagnóstico de discopatia. Revista de Medicina Desportiva, 5(3): 4-7.

Novoa, T. (2015). Relación entre el equilibrio y la práctica de surf en personas sanas Escuela de Fisioterapia. Universidad de Gambiadie - Cantabria. http://eugdspace.eug.es/xmlui/bitstream/ handle/123456789/277/Novoa Ortiz\%2C Tamara. pdf?sequence=1\&isAllowed=y

Paillard, T., Margnes, E., Portet, M. y Breucq, A. (2011). Postural ability reflects the athletic skill level of surfers. European Journal of Applied Physiology, 111(8), 1619-1623. https://doi.org/10.1007/ s00421-010-1782-2

Peirão, R., Tirloni, A. y Reis, D. (2008). Avaliação postural de surfistas profissionais utilizando o método Portland State University (PSU). Fitness \& Performance Journal, 7(6): 370-374. https://doi. org/10.3900/fpj.7.6.370.p

Slayback, B. (2014). Spinal cord injury and surfing: A quality of life study. Universidad del Estado de California. http://repositorio.unan.edu.ni/2986/1/5624.pdf

Wu, D. (2018). Online position recognition and correction method for sports athletes. Cognitive Systems Research, 52: 174-181. https://doi.org/10.1016/j.cogsys.2018.07.002 